\title{
Modeling of carrier dynamics in quantum-well electroabsorption modulators
}

\author{
Højfeldt, Sune; Mørk, Jesper
}

Published in:

I E E E Journal on Selected Topics in Quantum Electronics

Link to article, DOI:

10.1109/JSTQE.2002.806715

Publication date:

2002

Document Version

Publisher's PDF, also known as Version of record

Link back to DTU Orbit

Citation (APA):

Højfeldt, S., \& Mørk, J. (2002). Modeling of carrier dynamics in quantum-well electroabsorption modulators. I E E E Journal on Selected Topics in Quantum Electronics, 8(6), 1265-1276.

https://doi.org/10.1109/JSTQE.2002.806715

\section{General rights}

Copyright and moral rights for the publications made accessible in the public portal are retained by the authors and/or other copyright owners and it is a condition of accessing publications that users recognise and abide by the legal requirements associated with these rights.

- Users may download and print one copy of any publication from the public portal for the purpose of private study or research.

- You may not further distribute the material or use it for any profit-making activity or commercial gain

- You may freely distribute the URL identifying the publication in the public portal

If you believe that this document breaches copyright please contact us providing details, and we will remove access to the work immediately and investigate your claim. 


\title{
Modeling of Carrier Dynamics in Quantum-Well Electroabsorption Modulators
}

\author{
Sune Højfeldt and Jesper Mørk
}

\begin{abstract}
We present a comprehensive drift-diffusion-type electroabsorption modulator (EAM) model. The model allows us to investigate both steady-state properties and to follow the sweep-out of carriers after pulsed optical excitation. Furthermore, it allows for the investigation of the influence that various design parameters have on the device properties, in particular how they affect the carrier dynamics and the corresponding field dynamics. A number of different types of results are presented. We calculate absorption spectra and steady-state field screening due to carrier pile-up at the separate-confinement heterobarriers. We then move on to look at carrier sweep-out upon short-pulse optical excitation. For a structure with one well, we analyze how the well position affects the carrier sweep-out and the absorption recovery. We calculate the field dynamics in a multiquantum-well structure and discuss how the changes in the field near each well affect the escape of carriers from that well. Finally, we look at the influence that the separate-confinement heterostructure barriers have on the carrier sweep-out.
\end{abstract}

Index Terms-All-optical signal processing, device design, drift-diffusion model, electroabsorption modulator (EAM), optical communication, quantum-well devices, semiconductor devices, sweep-out dynamics.

\section{INTRODUCTION}

$\mathbf{S}$ OPHISTICATED COMPONENTS are required to realize both $40-\mathrm{Gb} / \mathrm{s}$ systems and next-generation all-optical systems. A device that is currently receiving much attention in relation to such systems is the electroabsorption modulator (EAM). Understanding its detailed dynamics can support the design of faster components and components that are tailored for specific functions, such as pulse generation or all-optical wavelength conversion.

The EAM has been used both for pulse generation [1] and to perform a number of all-optical functions such as demultiplexing [2], [3], wavelength conversion [4], and signal regeneration [5], [6] and may very well become an important part of future all-optical components.

All-optical functions are performed with EAMs by reducing the device absorption through optical excitation of carriers. There are two sources that contribute to the absorption change: Band filling and the quantum-confined Stark effect (QCSE). When carriers are excited into the wells, the absorption is reduced due to band filling. The excited carriers also affect the

Manuscript received September 3, 2002. This work was supported by the Danish Technical Research Council (STVF) through the SCOOP program.

The authors are with COM, Technical University of Denmark, DK-2800 Kgs.

Lyngby, Denmark (e-mail: sh@com.dtu.dk; jm@com.dtu.dk.)

Digital Object Identifier 10.1109/JSTQE.2002.806715

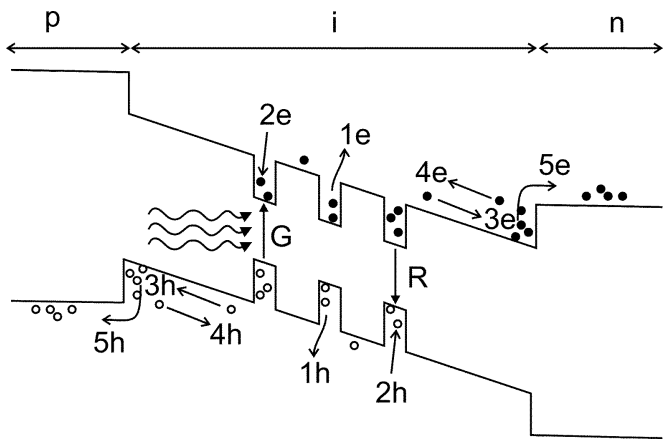

Fig. 1. Schematic illustration of a semiconductor EAM and the transport mechanisms that are used in PinSim to describe the carrier sweep-out dynamics.

field, and this leads to a change in the absorption through the QCSE.

In this paper, we investigate quantum-well EAMs. The dynamics of the carrier sweep-out process in an EAM is affected when carriers are excited in the device. Changes in the carrier mobilities and in the carrier escape times from the wells are induced by changes in the electric field. The pile-up of carriers at heterobarriers also affects the carrier sweep-out dynamics. When the density of photo-excited carriers is low, these changes will be of little importance. At higher densities, changes in the aforementioned parameters can significantly affect the dynamics. In particular, field-induced changes in the escape times of carriers out of the wells can increase the absorption recovery time compared to that in the low-density regime.

\section{MODEL}

Our model, PinSim, is in many ways similar to typical models for quantum-well semiconductor lasers [7]. We solve Poisson's equation and the continuity equations for electrons and holes, with the current densities being described by drift-diffusion equations. The bound states in the wells are found by solving Schrödinger's equation. The charge density represented by carriers in the bound states is included self-consistently into the Poisson and Schrödinger equations.

The carrier transport processes and transitions included in the model are shown in Fig. 1. They are $(\mathrm{G})$ carrier excitation, $(\mathrm{R})$ carrier recombination, (1) escape from wells, (2) capture into wells, (3) drift, (4) diffusion, and (5) thermionic emission across separate-confinement heterobarriers $(\mathrm{SCH})$.

In this paper, specifically in Sections III and IV, we investigate InGaAsP-based devices. 
TABLE I

PARAMETERS USED IN THE SiMULATIONS

\begin{tabular}{l|c}
\hline Parameter & Value \\
\hline Permittivity $\left[\epsilon_{0}\right]$ & 13.0 \\
Electron low-field mobility, $\mu_{\mathrm{n}}^{0}$ & 0.80 \\
{$\left[\mathrm{~m}^{2} / \mathrm{Vs}\right]$} & \\
Hole low-field mobility, $\mu_{\mathrm{p}}^{0}\left[\mathrm{~m}^{2} / \mathrm{Vs}\right]$ & 0.04 \\
Saturated velocity, electrons, $v_{\mathrm{nhf}}$ & $5.4 \cdot 10^{4}$ \\
{$[\mathrm{~m} / \mathrm{s}]$} & \\
Saturated velocity, holes, $v_{\mathrm{phf}}[\mathrm{m} / \mathrm{s}]$ & $5.2 \cdot 10^{4}$ \\
Electron velocity fitting parameter, & $0.8 \cdot 10^{-11}$ \\
$\beta\left[\mathrm{m}^{2} / \mathrm{V}^{2}\right]$ & \\
Matrix element, $|M|^{2}\left[e m_{0} / 2\right]$ & 24.0 \\
Refractive index, $n$ & 3.6 \\
\hline
\end{tabular}

\section{A. Poisson's Equation}

Poisson's equation relates the electrostatic potential $\phi$ to the charge densities

$$
\frac{d}{d x}\left(\epsilon \frac{d}{d x} \phi\right)=-e\left(p-n+N_{D}^{+}-N_{A}^{-}+p_{Q W}-n_{Q W}\right) .
$$

In this equation, $x$ is along the direction of growth, $\epsilon$ is the static permittivity (see Table I), $n$ and $p$ are the densities of electrons and holes, respectively, in bulk states, $N_{D}^{+}$and $N_{A}^{-}$are the densities of ionized donors and acceptors, respectively, and $n_{Q W}$ and $p_{Q W}$ are the charge distributions of electrons and holes, respectively, in the quantum wells. Finally, $e$ is the elementary charge.

\section{B. Bulk Continuity Equations}

The continuity equations for electrons and holes in bulk states are

$$
\begin{aligned}
\frac{\partial n}{\partial t} & =\frac{1}{e}\left(\frac{\partial J_{n}}{\partial x}+J_{c, Q W}^{3 D}\right)-R \\
-\frac{\partial p}{\partial t} & =\frac{1}{e}\left(\frac{\partial J_{p}}{\partial x}+J_{v, Q W}^{3 D}\right)+R
\end{aligned}
$$

where $J_{n}$ and $J_{p}$ are the electron and hole current densities and $R$ is the recombination rate. We assume that carriers are generated only in the wells, and thus there are no generation terms in the equations above. $J_{c, Q W}^{3 D}$ and $J_{v, Q W}^{3 D}$ are given by

$$
J_{\nu, Q W}^{3 D}(x)=\left\{\begin{array}{ll}
\frac{J_{\nu, Q W}^{k}}{L_{Q W}^{k}}, & x_{1}^{(k)}<x<x_{2}^{(k)} \\
0, & \text { otherwise }
\end{array}, \quad \nu=c, v .\right.
$$

The well width $L_{Q W}^{k}$ and the coordinates $x_{1}^{(k)}$ and $x_{2}^{(k)}$ that define the capture region for the $k$ th well are defined in Fig. 2. The two-dimensional (2-D) current densities $J_{\nu, Q W}^{k}(\nu=c, v)$ are calculated for each well individually. We return to these terms below, in Section II-D.

1) The Drift-Diffusion Equations: We use the drift-diffusion expressions

$$
J_{n}=e \mu_{n} n E+e D_{n} \frac{d n}{d x}, \quad J_{p}=e \mu_{p} p E-e D_{p} \frac{d p}{d x}
$$

for the current densities in (2) and (3). $E$ is the electric field, $\mu_{n}$ and $\mu_{p}$ are the field-dependent mobilities for electrons and

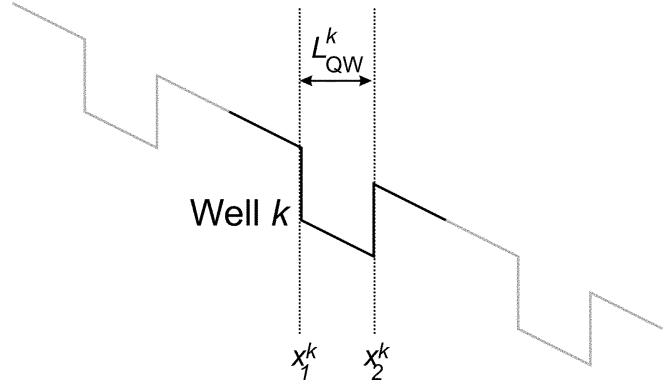

Fig. 2. Definitions of well width $L_{Q W}^{k}$ and the start and end coordinates $x_{1}^{(k)}$ and $x_{2}^{(k)}$, for the $k$ th well.

holes, respectively, and $D_{n}$ and $D_{p}$ are the diffusion parameters. The field-dependent mobilities are given by [8]

$$
\mu_{n}(E)=\frac{\mu_{n}^{0}+v_{\mathrm{nhf}} \beta|E|}{1+\beta E^{2}}, \quad \mu_{p}(E)=\frac{\mu_{p}^{0} v_{\mathrm{phf}}}{v_{\mathrm{phf}}+\mu_{p}^{0} E} .
$$

The parameters of these equations are taken from [8] and may be found in Table I. Lacking information on the diffusion parameters in the high-field regime, we use the Einstein relations

$$
D_{i}(E)=\frac{k_{B} T}{e} \mu_{i}(E), \quad i=n, p
$$

which are valid for a nondegenerate electron (hole) gas close to thermal equilibrium. The electric field is related to $\phi$ through

$$
E=-\frac{d \phi}{d x}
$$

\section{Quantum-Well Continuity Equations}

The continuity equations for the quantum wells are similar to those for carriers in bulk states. The change in the well carrier densities with time is accounted for by optical excitation and recombination and by exchange of carriers between well states and bulk states. For electrons and holes, respectively, in well $k$ we have

$$
\begin{aligned}
\frac{\partial n_{2 D}^{k}}{\partial t} & =-\frac{1}{e} J_{c, Q W}^{k}-R_{2 D}^{k}+G_{2 D}^{k} \\
\frac{\partial p_{2 D}^{k}}{\partial t} & =\frac{1}{e} J_{v, Q W}^{k}-R_{2 D}^{k}+G_{2 D}^{k}
\end{aligned}
$$

where $n_{2 D}^{k}$ and $p_{2 D}^{k}$ are the electron and hole densities, respectively, in well $k, J_{\nu, Q W}^{k}$ are the current densities of electrons $(\nu=c)$ and holes $(\nu=v)$ between the well states and the bulk states for well $k$; expressions for these terms may be found in Section II-D below. These terms also appear in the bulk continuity (2) and (3) through the expression in (4). $R_{2 D}^{k}$ is the recombination rate for carriers in well $k . G_{2 D}^{k}$ is the generation rate of optically excited carriers in well $k$

$$
G_{2 D}^{k}=-\alpha_{\mathrm{mat}}^{(k)} L_{Q W}^{k} v_{g} S
$$

where $v_{g}$ is the group velocity and $S$ is the photon density at well $k$, and $\alpha_{\text {mat }}^{(k)}$ is the material absorption for well $k$. The absorption is discussed in Section II-G. 


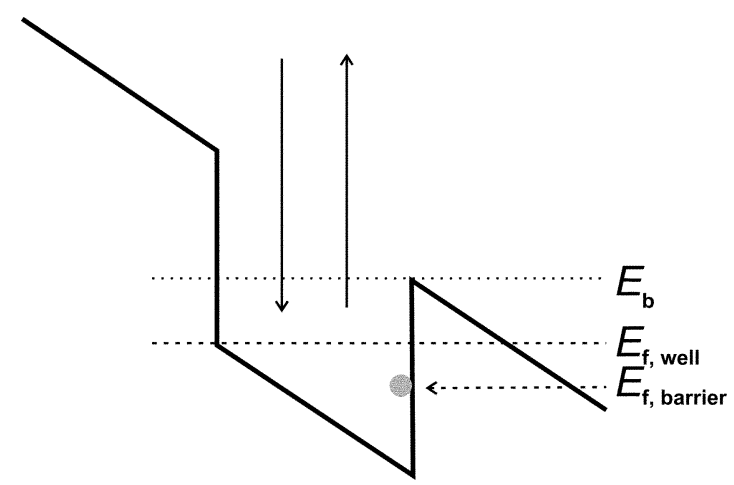

Fig. 3. Capture to and escape from a quantum well. The parameters are $E_{b}$, the barrier energy; $E_{f, \text { well }}$, the Fermi level in the well; and $E_{f \text {,barrier }}$, the Fermi level in the barrier. The grey dot indicates both the barrier Fermi level and the position at which it is measured.

\section{Carrier Transport Between Well and Barrier}

The transport of carriers out of and into the wells has a large influence on the speed of quantum-well EAMs. In a quantum-well semiconductor laser, deep wells are desirable because they have a higher density of carriers in the wells than do shallow wells. (The depth of the wells should of course not be so high that capture into the wells is impeded.) In a modulator, it is important to have a fast escape of carriers from the wells or the device will perform poorly at high speeds due to charge build-up in the wells. Short escape times are obtained by reducing the well depths or by increasing the field across them. However, reducing the well depths will reduce the electron-hole overlap at higher fields. Hence, a faster escape from the wells comes at the expense of a reduced field-induced change in the absorption [9].

In general, there can be a current both in and out of a quantum well, as illustrated in Fig. 3. The expression derived in [10] for the electron current density out of a quantum well is

$$
J_{e, Q W}^{\text {out }}=-e n^{*}\left(\frac{k_{B} T}{2 \pi m_{e, \|}}\right)^{1 / 2} \exp \left(-\frac{E_{b}-E_{1}}{k_{B} T}\right) .
$$

In this expression, $m_{e, \|}$ is the in-plane mass of electrons in the well, $n^{*}$ is the carrier density, and $E_{1}$ is the lowest lying quasi-bound state in the well. The masses used in this work are taken from [11]. We use $n^{*}=n_{2 D} / L_{\mathrm{QW}}$ where $L_{\mathrm{QW}}$ is the well width. $E_{b}$ is defined in Fig. 3. Calculated assuming a thermal distribution of carriers, this expression relies on fast carrier-carrier and carrier-phonon scattering. We also note that the expression is derived assuming that only the lowest well state is populated. We use an expression similar to (12) for the holes. Defining the escape time $\tau_{e, 2 D}$ through

$$
J_{e, \mathrm{QW}}^{\mathrm{out}}=\frac{n_{2 \mathrm{D}}}{\tau_{e, 2 \mathrm{D}}}
$$

we find

$$
\tau_{e, 2 \mathrm{D}}=\left(\frac{2 \pi m_{e, \|} L_{\mathrm{QW}}^{2}}{k_{B} T}\right)^{1 / 2} \exp \left[\frac{E_{b}-E_{1}}{k_{B} T}\right] .
$$

In this paper, we will use two different models for the capture of carriers into the wells. In the first model, we assume that there

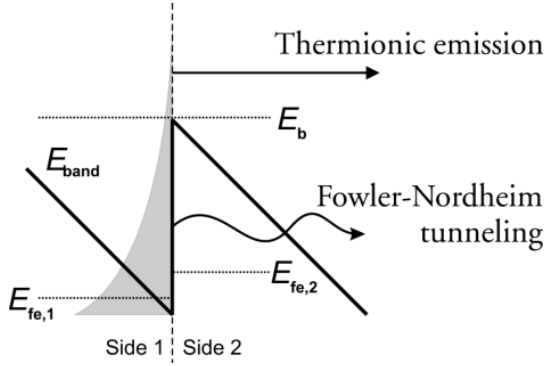

Fig. 4. Various mechanisms for transport across a heterobarrier. The gray area illustrates a thermal carrier distribution. The mechanisms are thermionic emission, where thermal carriers with high energies cross the barrier, and Fowler-Nordheim tunneling, where thermal carriers having energies lower than the barrier energy cross the barrier by tunneling through it. $E_{\mathrm{fe}, i}$ are the (electron) Fermi levels on the two sides of the junction, and $E_{b}$ is the barrier energy. $E_{\text {band }}$ is the band edge.

is a current density back into the well which ensures that the current density between the bulk and well states is zero in thermal equilibrium. The capture times thus determined are taken to be valid also away from equilibrium. The term "thermionic recapture" is used to refer to this model, which we use when we investigate steady-state properties. The reason for doing so is that the capture of carriers is a slow processes in EAMs, and only after a long time will the capture process establish a thermal equilibrium.

In the second model, we assume that there is no recapture of carriers into the wells. The term "no recapture" is used to refer to this model, which we use when we calculate the recovery after short-pulse excitation. As just mentioned, capture is a slow process, and thus the net current density out of the well will be determined by (12) after excitation with a short pulse. We will assume that this is true during the entire recovery, which takes a few hundred picoseconds or less in typical modulator structures.

\section{E. Transport Across Separate-Confinement Heterobarriers}

The transport across heterobarriers is dominated by thermionic emission, and we calculate this current using the expression given in [12]. For electrons, we have

$$
\begin{aligned}
& J_{e, \mathrm{th}}=-\frac{e \eta\left(m_{e, 1}, m_{e, 2}\right)\left(k_{B} T\right)^{2}}{2 \pi^{2} \hbar^{3}} \\
& \quad \cdot\left[\exp \left(-\frac{E_{b, e}-E_{\mathrm{fe}, 1}}{k_{B} T}\right)-\exp \left(-\frac{E_{b, e}-E_{\mathrm{fe}, 2}}{k_{B} T}\right)\right] .
\end{aligned}
$$

Here, $E_{\mathrm{fe}, i}$ are the Fermi levels on the two sides of the junction (see Fig. 4), $E_{b, e}$ is the barrier energy for electrons, and $m_{e, 1}$ and $m_{e, 2}$ are the electron masses on Side 1 and Side 2, respectively. The function $\eta$ is defined by [12]

$$
\eta\left(m_{1}, m_{2}\right)=\frac{2}{\frac{1}{m_{1}}+\frac{1}{m_{2}}} .
$$

For both hole types, which are assumed to have a common Fermi level, the current is determined by using similar expressions.

Another contribution to the transport across these barriers comes from Fowler-Nordheim tunneling, which is illustrated in Fig. 4. We calculate this contribution using expressions from [13]. 


\section{F. Well States}

In the common picture of optical excitation in EAMs, carriers are excited into confined well states from which they then escape. Thus, the picture assumes that for each well there is a set of localized states that are separated from the bulk states. This distinction allows us to find a separate Fermi level for each well, which means that we will be in a position to use the methods described above for calculating the escape of carriers out of each well and to calculate the spatial distribution of the carriers in the wells. This picture is used in the present work. Wanting to consider the wells individually, we divide the well region into individual cells. The potential is put to infinity at the midpoints of the barriers between wells, and the bound states for each of these special potentials are then found. This approach has been used extensively in modeling of absorption spectra and escape times from quantum wells in an electric field [14]-[16], also in laser simulators [17]. The more confined the states are, the better will the approximation we make by dividing the wells in this way be. This implies that the approximation is better at lower fields.

In PinSim, eigenstates $\psi_{n}$ and eigenenergies $\varepsilon_{n}$ are found in the envelope approximation by solving the single-particle Schrödinger equation

$$
\left[-\frac{\hbar^{2}}{2} \frac{d}{d x}\left(\frac{1}{m_{\perp}(x)} \frac{d}{d x}\right)+V(x)\right] \psi_{n}(x)=\varepsilon_{n} \psi_{n}(x)
$$

for each well using for the potential $V$, the special potential described above. In (17), $m_{\perp}$ is the mass in the growth direction and $\hbar$ is Planck's constant. The exchange-correlation potential (which gives rise to bandgap renormalization) and Coulomb interaction (which gives excitonic states) are not included.

The states found using this method are technically all bound because they are eigenstates with respect to the special potential functions described above. This means that coupling between wells, which is the basis for Wannier-Stark modulators [18], but also influences strongly the absorption in electroabsorption modulator structures with shallow wells and/or narrow barriers and cannot immediately be calculated with this model.

Although it is necessary to divide the well region into individual wells when we solve the combined Poisson equation/continuity equations/Schrödinger equation problem, PinSim can also be used to calculate separately the absorption spectra. This is done by using the potential for the entire well region instead of dividing it as described above. Examples of such calculations are reported in Section III-A.

\section{G. Absorption}

The absorption spectrum is calculated using the states found as described in Section II-F. The material absorption spectrum for each well is calculated as a sum of contributions from transitions between hole states and electron states. For well $k$,

$$
\begin{aligned}
\alpha_{\text {mat }}^{(k)}(\hbar \omega)= & \sum_{i, j} \frac{1}{L_{Q W}^{(k)}} \frac{1}{\hbar \omega} \frac{\pi e^{2} \hbar}{\epsilon_{0} c m_{0}^{2} n} M^{2} \frac{m_{r}}{2 \pi \hbar^{2}}\left|\left\langle F_{i} \mid G_{j}\right\rangle\right|^{2} \\
& \cdot\left(1-f_{c}-f_{v}\right) \cdot \Theta\left(\hbar \omega-\left(E_{c}^{(i)}-E_{v}^{(j)}\right)\right)
\end{aligned}
$$

The indices denote electron state $i$, with eigenenergy $E_{c}^{(i)}$ and hole state $j$, with eigenenergy $E_{v}^{(j)}$. $\Theta$ is the Heaviside function. (The Heaviside function is defined as

$$
\Theta(x)=\left\{\begin{array}{ll}
0, & x \leq 0 \\
1, & x>0
\end{array} .\right.
$$

$F_{i}$ is the normalized electron wavefunction, $G_{j}$ is the normalized hole wavefunction, $A \hat{\boldsymbol{e}}$ is the vector potential for the optical field ( $\hat{\boldsymbol{e}}$ is a unit vector), $M^{2}$ is the square of the momentum matrix element, and $n$ is the index of refraction (see Table I). The well index $k$ only appears on the absorption coefficients and on the well width, not on the other constituent parameters. However, those parameters may vary from one well to the next.

Equation (18) assumes that the subband dispersion is parabolic [19], [20]. The last term in the expression accounts for the occupation probability of carriers in the conduction band $f_{c}$ and in the hole band $f_{v}$. The reduced density-of-states mass $m_{r}$ is

$$
m_{r}=\left(\frac{1}{m_{e, \|}}+\frac{1}{m_{t h, \|}}\right)^{-1}
$$

where $m_{e, \|}$ is the in-plane electron mass and $m_{t h, \|}$ is the in-plane hole mass, either light $(t=l)$ or heavy hole $(t=h)$, depending on the hole type of eigenstate $j$ above. The light and heavy holes are assumed to be in a common thermal (quasi-)equilibrium.

The absorption coefficient of a well depends on the field across that well through the quantum-confined Stark effect. Because we calculate the absorption coefficient of each well dynamically, taking into account the instantaneous field across that well, this effect is included.

We have not included excitonic absorption, and thus we underestimate the absorption changes near the band edge.

The absorption change per well can be divided into two parts: One that is due to changes in the field, and one that is due to band filling. Before excitation, there are almost no carriers in the wells. If the density of carriers in a well is zero, and the field across the well is changed, the absorption spectrum will change. This change will be referred to as the field contribution to the absorption change. After excitation, there will be carriers in the well, and the presence of these carriers gives a further contribution to the absorption change. This contribution will be referred to as the band filling contribution to the absorption change. Formally, let $\alpha=\alpha(F(t), N(t))$ be the absorption coefficient at field $F(t)$ and let $N(t)$ be the carrier density for a given well. Before excitation (at $t=0 \mathrm{ps}$ ), there is some field $F_{0}$ across the well and a carrier density $N_{0}(\approx 0)$ in the well. The corresponding absorption coefficient is $\alpha\left(F_{0}, N_{0}\right)$. After optical excitation, the total absorption change at time $t$ is

$$
\Delta \alpha_{\text {total }}(t)=\left.\alpha\right|_{F(t), N(t)}-\left.\alpha\right|_{F_{0}, N_{0}} \text {. }
$$

The field contribution to the absorption change is

$$
\Delta \alpha_{\text {field }}(t)=\left.\alpha\right|_{F(t), N_{0}}-\left.\alpha\right|_{F_{0}, N_{0}}
$$

and the band filling contribution is

$$
\Delta \alpha_{\text {band-fill }}(t)=\left.\alpha\right|_{F(t), N(t)}-\left.\alpha\right|_{F(t), N_{0}} \text {. }
$$




\section{H. Boundary Conditions}

In the simulator, the electrostatic voltage across the structure, from contact to contact, is kept fixed (that is, the voltage source has zero internal resistance and is able to adjust the potential instantaneously). Furthermore, we assume that the electron and hole Fermi levels are equal at the boundaries of the structure, that is, that electrons and holes are in thermal equilibrium at the boundaries

$$
\begin{aligned}
& E_{f v}(x=0)=E_{f c}(x=0) \\
& E_{f v}(x=L)=E_{f c}(x=L) .
\end{aligned}
$$

The $\mathrm{p}$-contacting point is at $x=0$ and the $\mathrm{n}$-contacting point is at $x=L$.

\section{Steady-State Results}

This section presents calculations of steady-state properties for various epitaxial structures.

\section{A. Modeling of Absorption Spectra}

The absorption spectrum is obviously of the utmost importance in EAMs, whether they are driven optically or electrically. The change in absorption should be as large as possible for a given change in the applied bias. It is also important to minimize the insertion loss in the modulator's on-state (the low-absorption operation point), and one may be interested in designing a device with a low polarization-dependent loss. For all-optical applications, a device with a low polarization-dependent loss will be simpler to operate and will be more power-efficient than devices with nonnegligible polarization-dependent loss.

When the EAM is used as an external modulator, a low polarization-dependent loss may not be critical. Semiconductor lasers typically lase in only one polarization, and thus the design of a modulator for external modulation is subject to significantly relaxed conditions. A considerable amount of work has gone into designing structures with low polarization-dependent losses [21]-[25]. These structures are typically realized by using tensile strain in the wells to reduce the light hole bandgap (and using compressive strain in the barriers to avoid crystal dislocations).

Here, we consider a modulator structure designed for external modulation. The device has wells with a slightly compressive strain. Both TE and TM spectra were measured for the structure for a number of biases from 0 to $8 \mathrm{~V}$. Light from a tunable CW laser was coupled into the device using a fiber. The output light was collimated with a microscope objective, polarization-filtered, and then detected with a broad-area detector.

1) Band Diagram: The structure, which has ten wells, is shown in Fig. 5. The Tersoff band line-up model was used to calculate the band offsets [26], [27]. Note that the Tersoff line-up model yields electron wells that are significantly deeper than both types of hole wells. This is in stark contrast to the conventional constant-offset line-up model, which assumes a ratio between the conduction-band offset and the bandgap of around 0.4 [28], [29]. Because the mass of the electron is significantly lower than the mass of the heavy hole, modifying the structure in such a way that the electron well is deeper than the heavy-hole

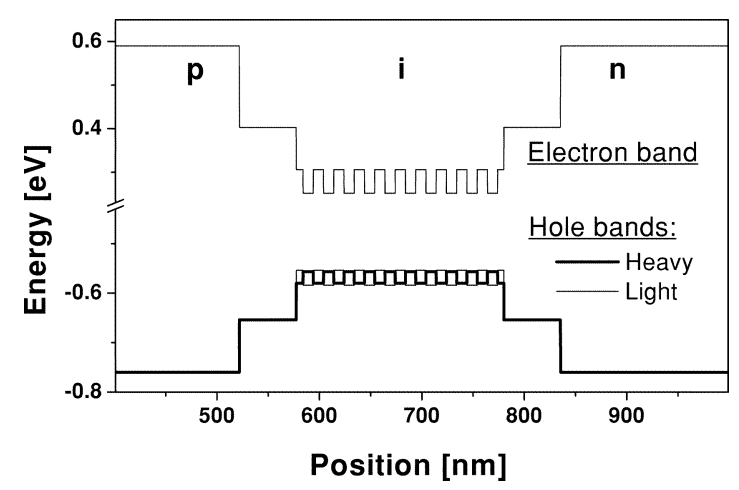

Fig. 5. Band diagram for the structure for which the absorption spectra are calculated.

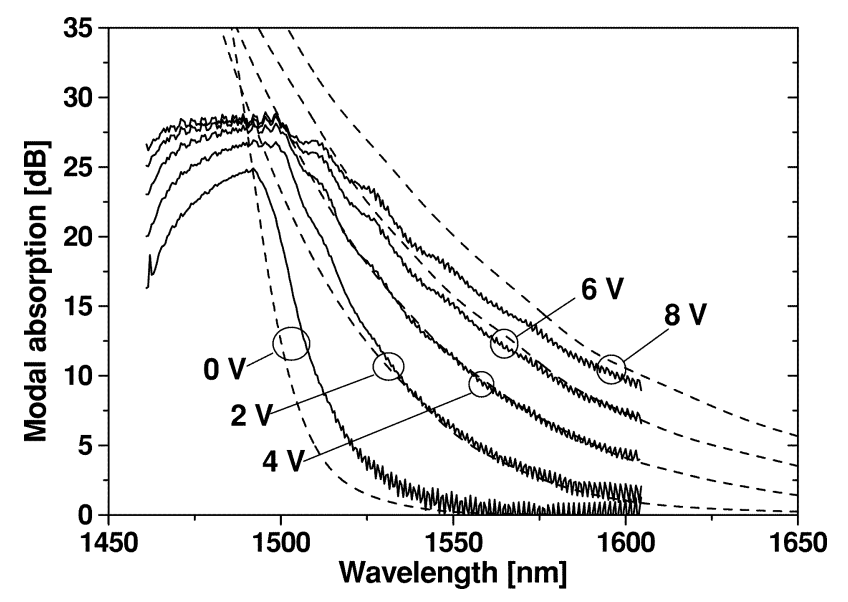

Fig. 6. Measured and calculated TE spectra for the structure shown in Fig. 5. The component is $250 \mu \mathrm{m}$ long. Note that we show the modal absorption.

well will result in a larger overlap between the ground state wavefunctions at low fields [9], [30].

The depths of the hole wells are only about the room-temperature thermal energy. The depth of the electron wells are a few times that.

2) Measured and Calculated Absorption Spectra: Fig. 6 shows the measured and calculated TE absorption spectra at various biases, and Fig. 7 shows the TM spectra. The device has a ridge waveguide, with a ridge width of $3 \mu \mathrm{m}$. The p-side is doped to $1.0 \cdot 10^{24} \mathrm{~m}^{-3}$, the n-side to $1.5 \cdot 10^{24} \mathrm{~m}^{-3}$.

The correspondence between the measurements and the calculations is quite good for both polarizations, with respect to both the wavelength dependence and the bias dependence. Both the wavelength dependence and the bias dependence of the TE and TM spectra are critically dependent on the band offsets, and we conclude that the band offsets calculated using the Tersoff line-up model approximate the real offsets well. The relation between the material absorption and the modal absorption includes the confinement factor, and the spectra presented above are calculated using a confinement factor $\Gamma=0.02$ for all wells and both polarizations, and using a device length of $250 \mu \mathrm{m}$. The confinement factor is estimated from an optical mode calculation.

Our calculated spectra show a larger modal absorption for the high energies than do the measured spectra. In Fig. 7, we have included in a simplified way a small contribution to the 


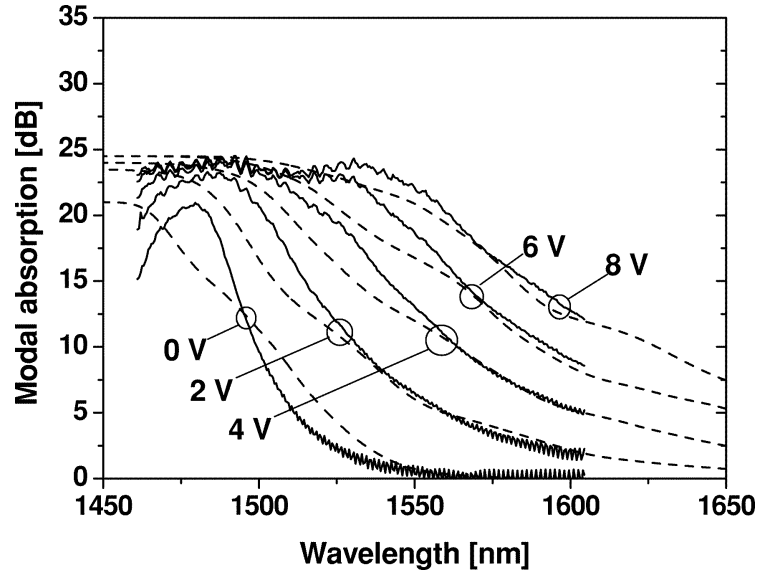

Fig. 7. Measured and calculated TE spectra for the structure shown in Fig. 5. The component is $250 \mu \mathrm{m}$ long. Note that we show the modal absorption.

transmission from a "first-order" mode that is assumed to not be absorbed. It is clear that including a higher order mode in this way enhances the correspondence at higher energies, as seen in Fig. 7, and such a mode might therefore, at least in part, be responsible for the behavior seen in the measured spectra.

The reason why the absorption in the measured spectra is reduced at higher energies is not known to us.

\section{B. Field Screening Due to Heterobarriers}

The separate-confinement heterostructure ( $\mathrm{SCH}$ ) in semiconductor waveguide-based lasers and modulators has an index-ofrefraction profile which provides guiding of light in modes with relatively low propagation losses. It also ensures a large coupling between the optical mode and the active region. However, the SCH hampers carrier escape in EAMs.

Typically, epitaxial InGaAsP structures are grown on InP. The bandgap of the seed-wafer material ( $\mathrm{InP})$ is $1.35 \mathrm{eV}$, whereas the active region usually is designed for operation at longer wavelengths, typically near $1550 \mathrm{~nm}$. The transition between the two bandgaps is typically made in a stepwise fashion because it is by far the simplest to realize when it comes to the InGaAsP material system.

The potential barriers incorporated into an epitaxial structure must be overcome by carriers on their way to the contacts. If a barrier is high compared to the thermal energy, carriers will pile up near it [31]-[33]. This pile-up is a problem in components that depend on changes in the electric field in the active region, such as the EAM. The carriers that are caught at the heterobarriers screen the field. Consequently, the field in the active region is affected when the optical power produces a large density of photo-excited carriers. Screening the field in the well region also reduces the escape rate of carriers out of the wells through (14).

The physics of this pile-up effect is similar to that of the thermionic emission model used for quantum wells and described in Section II-D. However, there is an important difference. For the bulk heterobarrier dynamics, it is not possible to attribute a simple time constant to the transport. In a sense, it is the direct-current properties that are affected by the height of the barriers. The same current density can be supported by a small or a large barrier, but the degree of field screening that results in the intrinsic region for a given current density will be

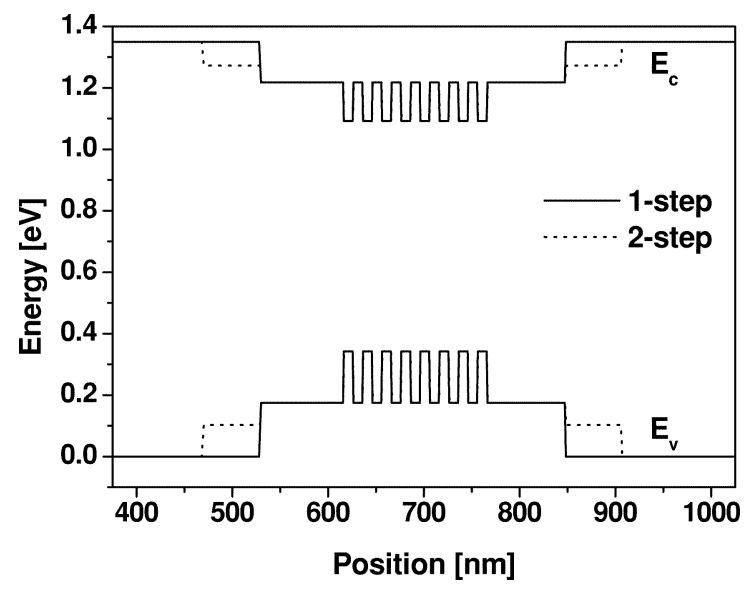

Fig. 8. Illustration of the two structures that are investigated to determine the influence of the heterobarrier design on the steady-state properties.

different for different barrier heights. The higher the barrier, the greater the pile-up.

To illustrate the influence of the heterobarrier structure on the power-handling capabilities, we examine how the field is screened in two structures with different heterobarrier structures. Fig. 8 shows the two different structures. We use a constant 43/57 line-up. The intrinsic regions in the two structures are the same, but one structure has a one-step barrier ("1-step" in Fig. 8) between the p-contact and the intrinsic region and between the intrinsic region and the n-contact; the second structure ("2-step" in Fig. 8) has two steps on both sides of the intrinsic region. Because the intrinsic-region widths in the two structures are different, we need to apply different voltages to the two structures to obtain the same field across their well regions before optical excitation. We do this to ensure that the mobilities and the times carriers take to escape from the wells are the same in the two cases. For the 1-step structure, the bias is $-0.3 \mathrm{~V}$; for the 2-step structure, it is $-0.62 \mathrm{~V}$. The structures are excited at $1600 \mathrm{~nm}$ with an optical power level of $2 \mathrm{~mW}$, and we have assumed thermionic recapture.

Fig. 9 shows the field distributions in the intrinsic regions of the two structures. Note that, for both structures, the outermost field changes are due to the transition from a highly doped region to an undoped region ("p-i" marks an interface between a p-doped region and an undoped region, "i-n" marks an interface between an n-doped region and an undoped region).

The difference is significant. More carriers pile up in the one-step structure, and this leads to a larger field screening in this structure than in the two-step structure. It can also be seen that holes contribute more to the screening than electrons.

For a given power level, the larger field screening in the one-step structure will result in a lower absorption coefficient in this structure than in the two-step structure. Because the carrier sweep-out rate is lower in the one-step structure under excitation, due to the larger field screening, the carrier excitation rate must also be lower. In quantitative terms, the absorption coefficient at zero excitation power is $9.16 \cdot 10^{3} \mathrm{~cm}^{-1}$ for both structures. The absorption coefficient at $2 \mathrm{~mW}$ is $5.64 \cdot 10^{3} \mathrm{~cm}^{-1}$ for the one-step structure and $6.23 \cdot 10^{3} \mathrm{~cm}^{-1}$ for the two-step structure. It is worth noting that the $\sim 10 \%$ difference between the absorption coefficients is similar to the 


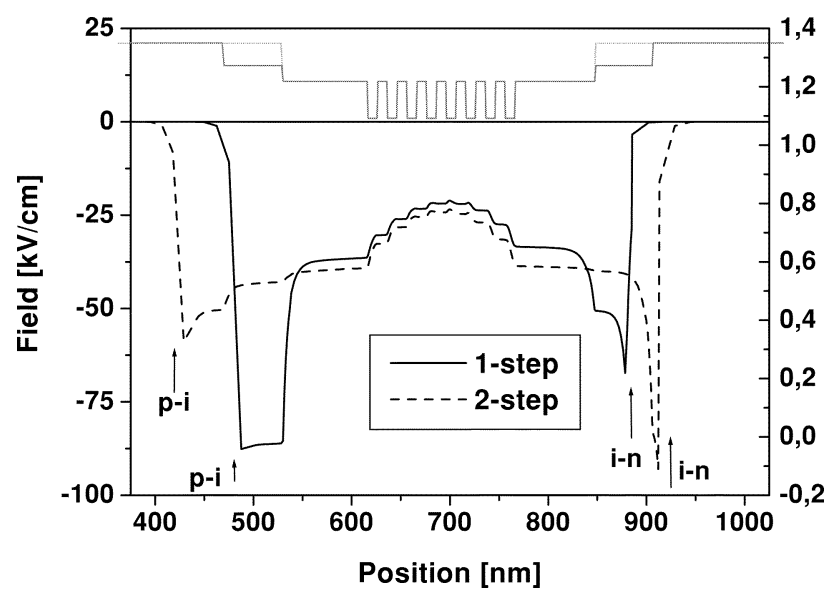

Fig. 9. Field distributions in the two structures shown in Fig. 8 under optical excitation. The excitation power level is $2 \mathrm{~mW}$. The band diagrams for the two structures are also shown.

difference between the fields in the well regions of the two structures, as shown in Fig. 9.

Our results clearly show that the heterobarriers must be given serious consideration when modulators are being designed. The screening of the field causes an increase in the escape times from the wells and thus reduces the speed of the component. A number of papers investigate carrier pile-up at heterointerfaces experimentally and show that dividing the barriers into several steps reduces carrier pile-up [31]-[33]. The calculations presented above quantify the pile-up in the steady state for two specific structures subjects to certain external conditions (bias, power level, excitation wavelength).

As shown above, dividing the single barriers in the one-step structure into two steps reduces the field screening significantly. Further calculations have shown that dividing the barriers into more than two steps reduces the field screening only a little further. From a wafer-growth perspective, this means that, although attention must be given to the design of the heterobarriers, a few barrier steps will often suffice. We investigate the influence of pile-up under pulsed excitation in Section IV-C.

A final point we wish to make here is that carrier pile-up decreases if the diffusion terms in (5) should become less important relative to the drift terms. This can be explained by the following argument (given for a single carrier type). If the diffusion term were reduced but the same number of carriers were present near the heterobarrier, then those carriers would be concentrated closer to the interface. This would increase the thermionic current across the barrier. Thus, equivalently, the density of piled-up carriers will be lower if the relative importance of the diffusion term is reduced.

The actual value of the diffusion constant, and its dependence on carrier density, field, and other variables, is very difficult to evaluate. The relation between diffusion and pile-up discussed above should therefore be considered a qualitative rather than a quantitative relation.

\section{Time-Resolved CARrier SweEP-Out Dynamics}

This section presents calculations of the carrier sweep-out in a number of different structures.

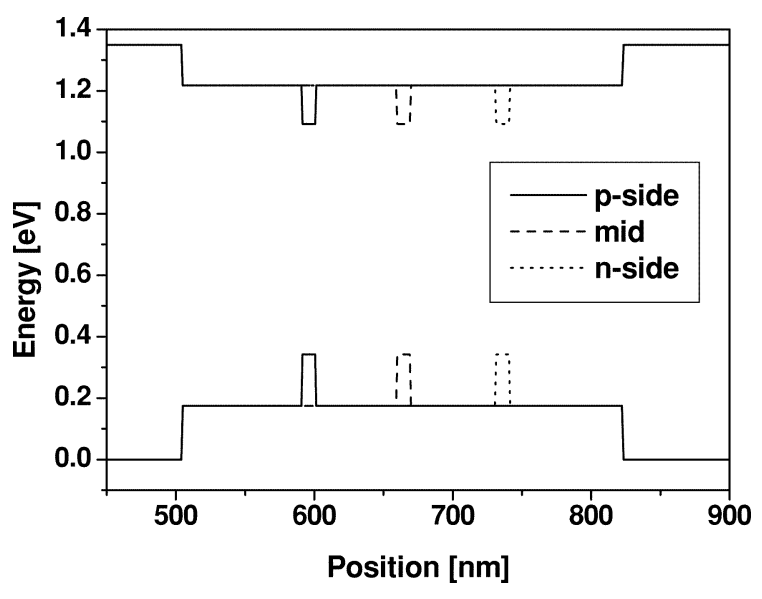

Fig. 10. Band diagrams for "p-side," "mid," and "n-side" structures, all of which have one well. The width of the intrinsic region is $320 \mathrm{~nm}$ in all cases. The distance from the middle of the well to the p-side barrier is 90,160 , and $230 \mathrm{~nm}$, respectively.

In all the calculations, a Gaussian, 1-ps (full-width at halfmaximum, FWHM) optical pulse centered at $t=2.5 \mathrm{ps}$ is used to excite the structures.

\section{A. Structures With One Well}

In this section, we investigate how a change in the position of a well influences the sweep-out dynamics. Changing a well's position affects the sweep-out because the distances to the two contacts change. Simulations of the sweep-out in structures with one well placed at various points in the intrinsic region can thus provide information about the bulk transport. They can also provide information about how the induced absorption change depends on the well position.

In the following, three structures are considered. They are unstrained, with $\operatorname{In}_{0.79} \mathrm{Ga}_{0.21} \mathrm{As}_{0.45} \mathrm{P}_{0.55}$ barriers and $\mathrm{In}_{0.53} \mathrm{Ga}_{0.47}$ As wells. Fig. 10 shows the band diagrams for the three structures. The nominal width of the intrinsic region is $320 \mathrm{~nm}$ for all the structures. We use a band line-up model in which the splitting $E_{c} / E_{v}$ between the conduction band offset and the valence band offset is $43 / 57$. The depth of the electron well is $126 \mathrm{meV}$, and the depth of both hole wells is $167 \mathrm{meV}$. The bandgap energy in the structures is $0.75 \mathrm{eV}(1650 \mathrm{~nm})$. The bias is $-0.27 \mathrm{~V}$ in all cases, and the structures are pumped and probed at $1600 \mathrm{~nm}$. Because the width of the intrinsic region is the same in all three structures, the field across the intrinsic regions will also be the same before excitation. Consequently, the absorption coefficient, the escape times from the wells, and the drift velocities are the same in all three structures before excitation. The excitation pulse energy is $0.5 \mathrm{pJ}$, which gives a carrier density of $\approx 1.5 \cdot 10^{15} \mathrm{~m}^{-2}$ in the wells. We have assumed that there is no recapture of carriers.

Fig. 11 shows, on the left, the absorption recovery in the three structures; in the middle, the field contributions to the absorption changes; and on the right, the band filling contributions to the absorption changes. The behavior of the total absorption recovery is very different in the three structures, whereas the band filling contributions to the absorption changes are very similar. The differences in the total absorption change for the three structures are therefore mostly due to the field contributions. 


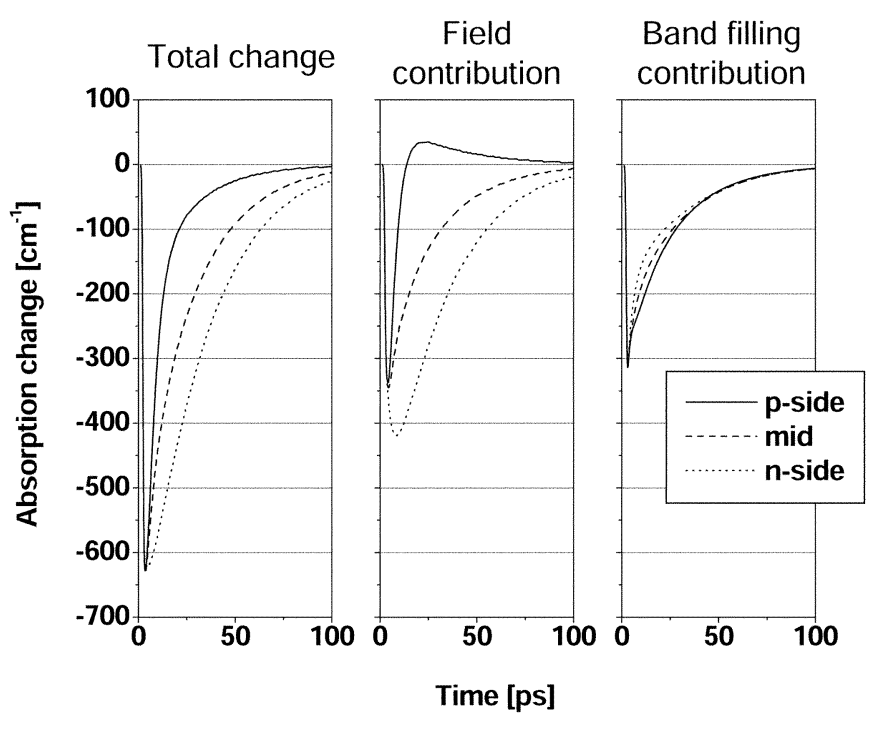

Fig. 11. Absorption changes in the three one-well structures with different positions of the well. The field and band filling contributions are also shown. The excitation pulse energy is $0.5 \mathrm{pJ}$ for all three structures.

The differences seen in the band filling contributions in the three structures (right-hand side of Fig. 11) result because the field changes across the wells are different in the three structures. These field changes are shown in Fig. 12. The field changes affect the escape times for holes and electrons (which are 25.7 and $3.4 \mathrm{ps}$, respectively, before excitation), and they affect the densities of states. The background for the differences in the field changes will be discussed below.

The absorption recovers faster in the "p-side" structure than in the other structures. From Fig. 11, it is clear that this is because the band filling contribution and the field contribution to the absorption change conspire to give an apparently faster recovery for this structure. However, looking at the band filling contributions, it is seen that the escape of carriers from the well in the three structures is about the same. This means that in a systems application, where the excitation is repetitive and carriers thus can accumulate, the three structures will operate up to approximately the same maximum bit rate. However, up to this rate, the p-side structure will provide the shortest switching window and will thus be preferable.

The electrostatic potential is assumed to be constant across the diode (see Section II-H). Thus, if $E(x ; t=0)$ is the field distribution before excitation and $E(x ; t)$ is the distribution at time $t$, then

$$
\int_{0}^{L}[E(x ; t)-E(x ; t=0)] d x=0
$$

where the limits of integration mark the $\mathrm{p}$-contacting point $(x=$ $0)$ and n-contacting $(x=L)$ point of the epitaxial structure. Since holes escape more slowly from the well than electrons, there will be a positive charge-density contribution at the well, and this will induce a change in the field through (1). On either side of the well, the field must adjust to ensure that (25) is fulfilled.

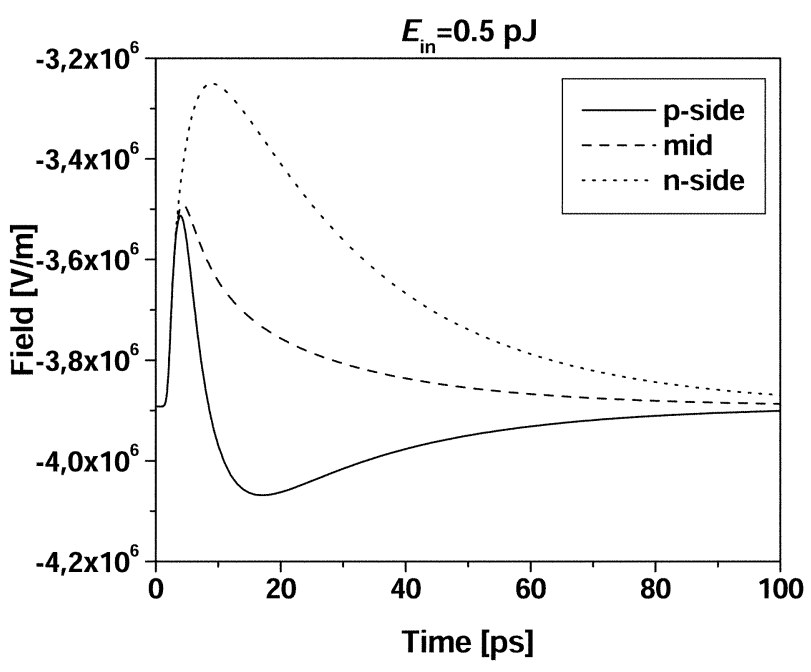

Fig. 12. Recovery of the field across the well in the three one-well structures with different positions of the well.

For the argument below, we will assume that the field is constant on both sides of the well (see Fig. 12) and denote by $\Delta E$ the field change induced by the carriers in the well. This field change is almost the same in the three structures, at least before the carriers reach the contacts. The change $\Delta E$ is split with a fraction $s$ on the p-contact side of the well and $(1-s)$ on the $\mathrm{n}$-contact side of the well. Because we have assumed that the field is constant on both sides of the well, we can approximate (25) by

$$
d_{p} s \Delta E-d_{n}(1-s) \Delta E=0
$$

where $d_{p}$ is the distance from the well to the p-contact and $d_{n}$ is the distance to the n-contact. From (26) we find

$$
s=\frac{d_{n}}{d_{p}+d_{n}} .
$$

Depending on the position of the well, the field on either side will change according to this ratio. In the "p-side" structure, where the well is closer to the p-side, we have $s=d_{n} /\left(d_{p}+\right.$ $\left.d_{n}\right)>1 / 2$ and thus the field change on the p-contact side of the well is larger than the field change on the n-contact side of the well and vice versa for the "n-side" structure. The actual field distributions before excitation and at $t=8 \mathrm{ps}$ are shown in Fig. 13. The arrows show the position of the well in each structure. The average field across the well in each structure can be seen in Fig. 12.

The argument above explains why the field changes across the wells are different for the three structures (keep in mind that $\Delta E$ is almost the same in the three structures). It implies that, if the electron escape time were longer than the hole escape time, the field changes would have been reversed (in a qualitative, not quantitative, sense) compared to those shown in Fig. 12: the field on the p-contact side of the well would have been reduced, and the field on the n-contact side would have increased. 


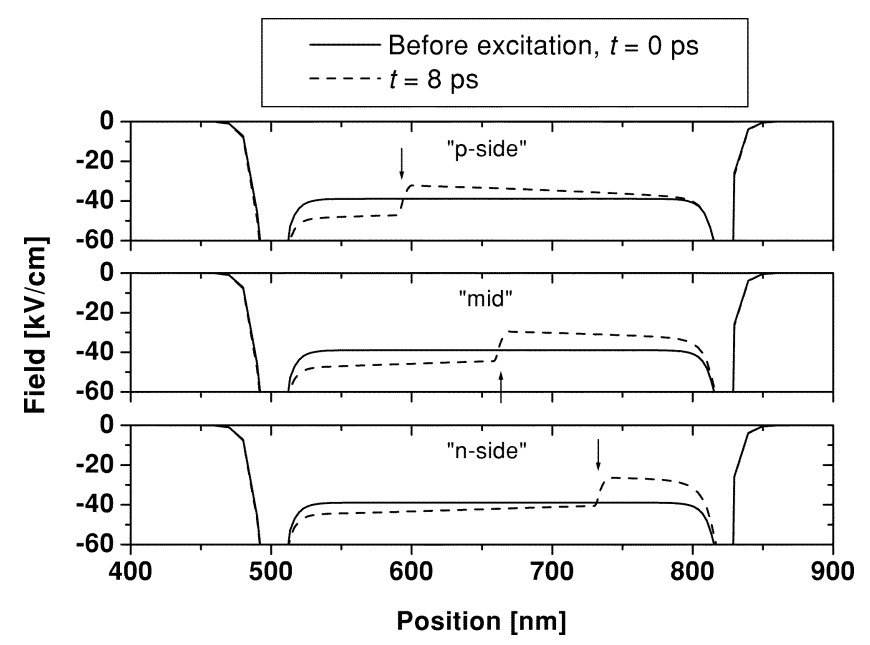

Fig. 13. Field distributions in the three one-well structures with different positions of the well. The fields are shown for times $t=0 \mathrm{ps}$ (that is, before excitation) and at $t=8 \mathrm{ps}$. The arrows mark the position of the well in the structures.

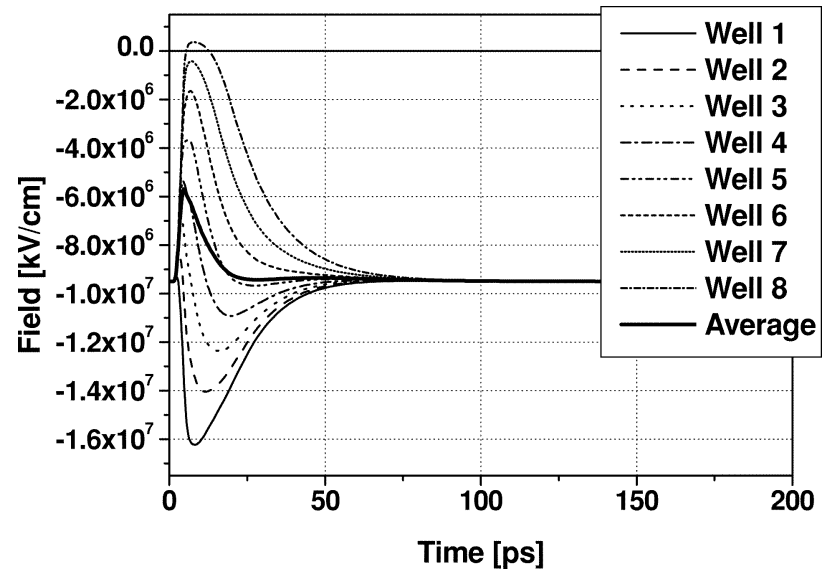

Fig. 14. The field across each well in our eight-well structure excited by a $0.5-\mathrm{pJ}$ pulse. The thick line is the average field.

\section{B. Detailed Dynamics in a Multiquantum-Well Structure}

In this section, we investigate the distributed nature of the sweep-out in structures that have more than one well. A structure with eight wells is considered. The structure is the one-step structure investigated in Section III-B, and its band diagram is shown in Fig. 8 (with label "1-step"). Except for its number of wells, it is similar to the structures from Section IV-A. The applied bias is $-2 \mathrm{~V}$, and the absorption is pumped at $1600 \mathrm{~nm}$. We assume that there is no recapture.

Fig. 14 shows the field across each of the eight wells after excitation with a $0.5-\mathrm{pJ}$ pulse. The wells are numbered starting from the p-contact side of the well region. As expected, the field change is different across the different wells. The essence of this behavior was discussed in Section IV-A. Adding seven extra wells is of course not a trivial change to a one-well structure. Still, the behavior for each of the wells is in qualitative agreement with what would be expected from the one-well simulations when each well's position is taken into account.

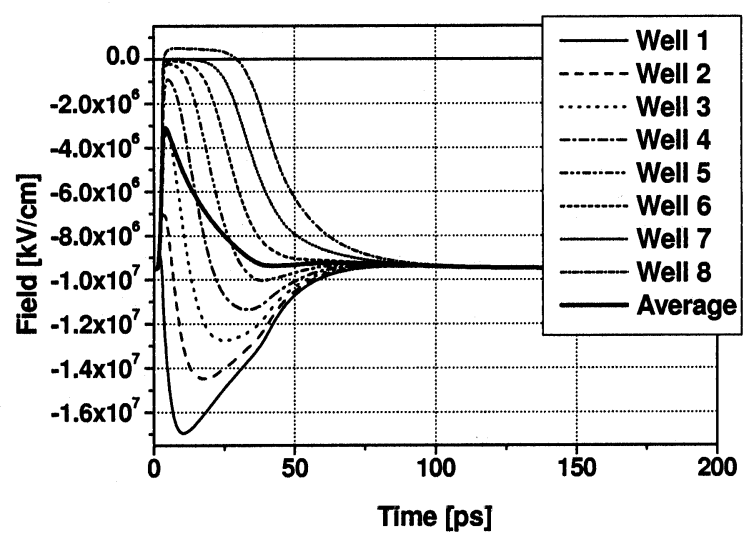

Fig. 15. The field across each well in our eight-well structure excited by a 5-pJ pulse. The thick line is the average field.

Fig. 14 also shows the average field across the entire well region. Not surprisingly, the average field experiences a decrease. However, if the wells were moved toward the p-contact, more wells would experience a field increase, and the average field would be reduced less and might even increase (we refer once again to Section IV-A). A field increase is not desirable for switching, where an absorption bleaching is sought. Moving the wells toward the n-contact instead would increase the field screening across the wells. This consideration is clearly important in the design of devices, especially devices for all-optical signal processing.

For voltage-modulation of the absorption, it might be desirable to move the wells closer to the p-side. When the field is high, the device has a large absorption (through the quantumconfined Stark effect). When carriers are excited, the field reduction in the well region will be smaller if the wells are closer to the p-side. As a consequence, the absorption reduction induced by the carriers through screening will also be smaller and might even increase as illustrated by Fig. 11. A reduced field screening is also advantageous because it reduces the field-induced increases of the escape times (from the wells, that is). Note that moving the wells around will affect the overlap between the optical field and the active region.

Fig. 15 shows the field change upon excitation with a 5-pJ pulse. The qualitative behavior is similar to that in Fig. 14. However, the 5-pJ pulse excites a higher density of carriers, thereby causing a larger field screening and also a larger average field screening. Fig. 15 also clearly shows that the carrier sweep-out takes longer. For Well 8, for instance, the field remains screened for a significantly longer time than after excitation with the $0.5-\mathrm{pJ}$ pulse.

For each of the two pulse energies, the difference in the carrier escape times from well to well is due to field screening. As shown by Figs. 14 and 15, the field increases at Well 1 (the well closest to the p-contact), changing gradually toward Well 8 , where it decreases. The changes in the field modify the carrier escape times as shown in Fig. 16 for the 0.5 -pJ case. Fig. 16 also illustrates the important fact that the escape time from a well increases faster when the field is screened than it decreases when the field is increased. Formally, $-d \tau_{\text {esc }} / d F$ decreases 

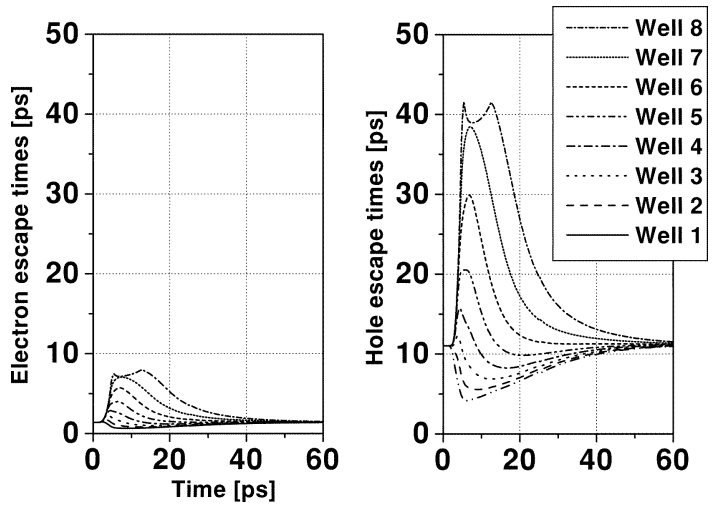

Fig. 16. Escape times for electrons and holes. The excitation pulse energy is $0.5 \mathrm{pJ}$.

with field, $F$; $\tau_{\text {esc }}$ is the escape time out of the well. This behavior is due to the exponential function in (14), which may be approximated as

$$
\exp \left[\frac{E_{b}-E_{1}}{k_{B} T}\right] \approx \exp \left[\frac{\frac{F L_{Q W}}{2}-\left(E_{b}-E_{1}^{(0)}\right)}{k_{B} T}\right]
$$

where $E_{1}^{(0)}$ is the eigenenergy of the lowest bound state at zero field. The calculations in this section, beside showing the nonuniformity in the field change across the different wells, also illustrate how the dynamical parameters, such as the escape times from the wells, can be affected by optical excitation. If the field across a well is screened substantially, the escape from that well can significantly reduce the device speed. This point emphasizes the importance of considering carefully how the wells are placed within the intrinsic region.

\section{Dynamics at Heterojunctions}

As discussed in Section III-B, heterobarriers affect the dynamics by causing a pile-up of carriers, which then screen the field.

In this section, the sweep-out upon short-pulse excitation is investigated for the two structures from Section III-B under the same bias conditions. The devices are pumped at $1570 \mathrm{~nm}$, and the excitation energy is $50 \mathrm{fJ}$.

Fig. 17 shows the field recovery in the two structures after excitation. The field screening persists for much longer in the one-step structure than in the two-step structure. Fig. 18 shows for various times the hole and electron densities in the intrinsic regions of the two structures. It is clear that the pile-up is much larger in the one-step structure than in the two-step structure. The long recovery time in the one-step structure is mainly due to holes, which pile up at the barrier close to the p-contact. Electrons also contribute to the screening, but less so. In the two-step structure there is only a negligible pile-up. It is important to note that holes and electrons escape from the wells with different characteristic times. Thus, the hole pile-up comes about later than the electron pile-up, and the time dependence of the pile-ups should therefore be considered independently for the two carrier types.

The widths of the intrinsic regions in the two structures are different. However, the intrinsic region in the two-step structure

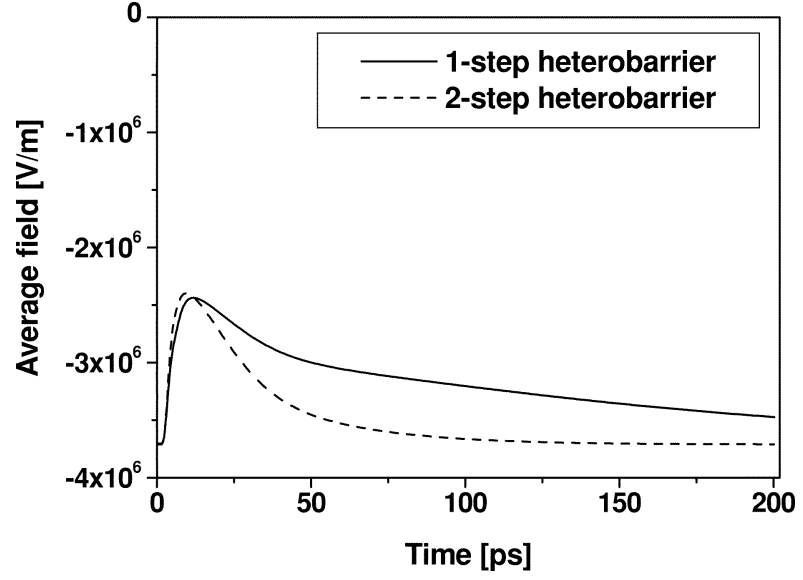

Fig. 17. Field recovery after optical excitation in the two structures shown in Fig. 8.

$\underline{1 \text {-step barriers }}$
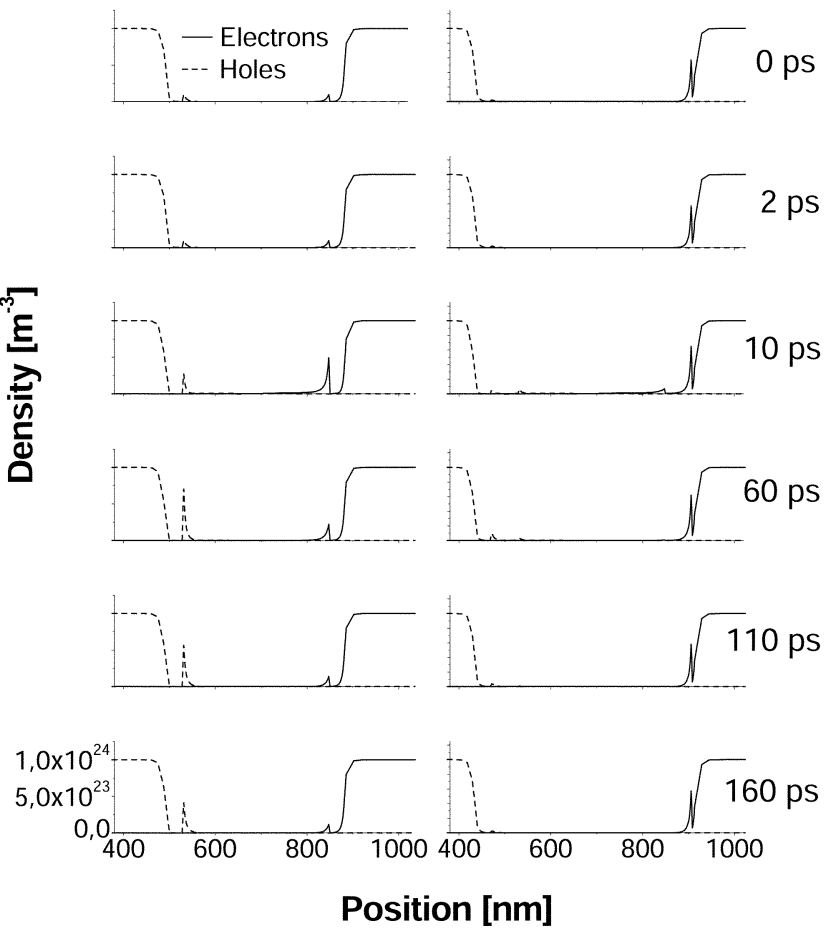

Fig. 18. Electron and hole densities across the intrinsic regions in the one-step and two-step structures at various times.

is only about $100 \mathrm{~nm}$ wider than that in the one-step structure, so the differences in carrier transport times to the heterojuntions are only a few picoseconds and thus are responsible for only an insignificant part of the differences seen in Fig. 17.

The pile-up decreases when the bias is increased [see (15)]. From an application point of view, it is important to investigate how a specific design affects the operation. For electrical modulation, for instance, the applied bias is typically relatively low because the absorption band edge is steeper at smaller fields. This reduces the voltage swing required for operation, but the low bias could result in carrier pile-up.

For operation at very high speed, the dynamics at the heterojunctions must be examined closely. Even slight charging effects 
will reduce the device response time. Division of the heterobarriers into more steps than two should be considered if the response time is being limited by the transport dynamics at the SCHs.

\section{CONCLUSION}

Using a comprehensive drift-diffusion model, we have investigated the carrier sweep-out dynamics in EAMs and its influence on the absorption dynamics.

The paper has presented examples of the kinds of results that can be investigated with the model. Measured absorption spectra were compared to spectra calculated with the model, and we found a fair agreement between the two. The influence of the $\mathrm{SCH}$ design on the steady-state field screening and dynamical sweep-out was investigated. In line with experimental results presented by other groups, we found that dividing one large step into two smaller steps can significantly reduce the pile-up in the steady state. Introducing more steps than two made only a small difference. Furthermore, the dynamical sweep-out in a structure with two steps will proceed significantly faster than in a structure with just one step.

Three structures, each having one well, were compared. We found that the absorption and field dynamics depend strongly on the well's position. However, the maximum bit rate at which the three devices can operate is nearly the same, limited by the sweep-out of holes from the wells. Below this bit rate, the "p-side" structure will be preferable as it provides the shortest switching window. The influence that the a well's position has on the field change it experiences was analyzed. Besides giving a general description of this relationship, the analysis allowed us to explain why the "p-side" structure provides the shortest switching window.

In accordance with these results, we found that the field across the different wells in a multiquantum-well structure is highly nonuniform. This influences the QCSE-induced change in the absorption for the different wells. Furthermore, the nonuniform behavior leads to nonuniform changes in the escape times for the different wells. The calculations therefore also provided an example of the complexity of the sweep-out process, demonstrating how optical excitation can affect the dynamical parameters such as the escape times from the wells.

\section{ACKNOWLEDGMENT}

The structure shown in Fig. 5 was designed in collaboration with K. Yvind (COM) who also grew the structure. K. Yvind (COM) also provided the parameterization of the band line-up model used in Section III-A1. The measurements were made by F. Romstad (COM). Both are thanked for fruitful discussions. The authors would also like to acknowledge the collaboration with GiGA - An Intel Company.

\section{REFERENCES}

[1] T. Ido, S. Tanaka, M. Suzuki, and H. Inoue, "MQW electroabsorption optical modulator for $40 \mathrm{Gbit} / \mathrm{s}$ modulation," Electron. Lett., vol. 31, no. 24, pp. 2124-2125, 1995 .
[2] A. D. Ellis, J. K. Lucek, D. Pitcher, D. G. Moodie, and D. Cotter, "Full $10 \times 10 \mathrm{Gbit} / \mathrm{s}$ OTDM data generation and demultiplexing using electroabsorption modulators," Electron. Lett., vol. 34, no. 18, pp. 1766-1767, 1998.

[3] J. Yu, K. Kojima, N. Chaud, M. C. Fischer, R. Espindola, and T. G. B. Mason, " $160 \mathrm{~Gb} / \mathrm{s}$ single-channel unrepeatered transmission over 200 km of nonzero dispersion shifted fiber," in Proc. ECOC 2001, Madrid, Spain, 2001, pp. 20-21.

[4] N. Edagawa, M. Suzuki, and S. Yamamoto, "Novel wavelength converter using an electroabsorption modulator," IEICE Trans. Electron. vol. E81-C, no. 8, pp. 1251-1257, 1998.

[5] T. Otani, T. Miyazaki, and S. Yamamoto, "40-Gb/s optical 3R regenerator using electroabsorption modulators for optical networks," J. Lightwave Technol., vol. 20, pp. 195-200, Feb. 2002.

[6] L. K. Oxenløwe, E. Hilliger, A. Tersigni, A. M. Nik, S. Højfeldt, F. Romstad, K. Yvind, P. M. W. Skovgaard, K. Hoppe, and J. Hanberg, "All-optical demultiplexing and wavelength conversion in an electroabsorption modulator," Proc. ECOC'01, pp. 604-605, 2001.

[7] N. Tessler, R. Nagar, G. Eisenstein, S. Chandrasekhar, C. H. Joyner, A. G. Dental, J. Koren, and G. Raybon, "Nonequilibrium effects in quantum well lasers," Appl. Phys. Lett., vol. 61, no. 20, pp. 2383-2385, 1992.

[8] K. J. Williams, R. D. Esman, and M. Dagenais, "Nonlinearities in P-I-N microwave photodetectors," J. Lightwave Technol., vol. 14, pp. 84-96, Jan. 1996.

[9] R. Y.-F. Yip, P. Desjardins, L. Isnard, A. Ait-Ouali, H. Marchand, J. L. Brebner, J. F. Currie, and R. A. Masut, "Band alignment engineering for high speed, low drive field quantum-confined Stark effect devices," $J$. Appl. Phys., vol. 83, no. 3, pp. 1758-1169, 1998.

[10] H. Schneider and K. von Klitzing, "Thermionic emission and Gaussian transport of holes in a GaAs/ $\mathrm{Al}_{x} \mathrm{Ga}_{1-x}$ As multiple-quantum-well structure," Phys. Rev. B, vol. 38, no. 9, pp. 6160-5, 1988.

[11] S. L. Chuang, Physics of Optoelectronic Devices. New York: Wiley, 1995.

[12] M. Grupen, K. Hess, and G. H. Song, "Simulation of transport over heterojunctions," in Proc. 4th Int. Conf. Simul. Semicon. Dev. Process, vol. 4, 1991, pp. 303-311.

[13] M. Lenzlinger and E. H. Snow, "Fowler-Nordheim tunneling into thermally grown $\mathrm{SiO}_{2}$," J. Appl. Phys., vol. 40, no. 1, pp. 278-83, 1969.

[14] D. A. B. Miller, D. S. Chemla, T. C. Damen, A. C. Gossard, W. Wiegmann, T. H. Wood, and C. A. Burrus, "Electric field dependence of optical absorption near the band gap of quantum-well structures," Phys. Rev. B, vol. 32, no. 2, pp. 1043-1060, 1985.

[15] P. J. Stevens, M. Whitehead, G. Parry, and K. Woodridge, "Computer modeling of the electric field dependent absorption spectrum of multiple quantum well material," IEEE J. Quantum Electron., vol. 24, pp. 2007-2016, Oct. 1988.

[16] B. Jonsson and S. T. Eng, "Solving the Schrodinger equation in arbitrary quantum-well potential profiles using the transfer matrix method," IEEE J. Quantum Electron., vol. 26, pp. 2025-35, Nov. 1990.

[17] M. Grupen and K. Hess, "Simulation of carrier transport and nonlinearities in quantum-well laser diodes," IEEE J. Quantum Electron., vol. 34 pp. 120-140, Jan. 1998

[18] H. Mimura, M. Hosoda, K. Tominaga, T. Watanabe, and K. Fujiwara, "Absorption saturation mechanism in short-period GaAs/AlAs superlattice self-electro-optic effect devices based on Wannier-Stark localization," Solid State Electron, vol. 40, no. 1-8, pp. 171-174, 1996.

[19] P. S. Zory Jr, Ed., Quantum Well Lasers. San Diego, CA: Academic, 1993.

[20] P. Blood, "On the dimensionality of optical absorption, gain, and recombination in quantum-confined structures," IEEE J. Quantum Electron., vol. 36, pp. 354-362, Mar. 2000.

[21] F. Devaux, S. Chelles, A. Ougazzaden, A. Mircea, M. Carré, F. Huet, A. Carenco, Y. Sorel, J. F. Kerdiles, and M. Henry, "Full polarization insensitivity of a $20 \mathrm{~Gb} / \mathrm{s}$ strained-MQW electroabsorptin modulator," IEEE Photon. Technol. Lett., vol. 6, pp. 1203-1206, Oct. 1994.

[22] K. Yamada, H. Murai, K. Nakamura, H. Satoh, Y. Ozeki, and Y. Ogawa, "10-Gbit/s EA modulator module with a polarization dependence of less than 0.3 dB," in Proc. OFC'95 Optical Fiber Communication, vol. 8, 1995 Technical Digest Series, 1995, pp. 24-25.

[23] A. Ougazzaden and F. Devaux, "Strained InGaAsP/InGaAsP/InAsP multi-quantum well structure for polarization insensitive electrobsorption modulator with high power saturation," J. Appl. Phys., vol. 69, no. 27, pp. 4131-4132, 1996.

[24] T. Ido, M. Koizumi, S. Tanaka, M. Suzuki, and H. Inoue, "Polarization and wavelength insensitive MQW electroabsorption optical gates for WDM switching systems," IEEE Photon. Technol. Lett., vol. 8, pp. 788-90, June 1996 
[25] D. G. Moodie, P. J. Cannard, A. J. Dann, D. D. Marcenac, C. W. Ford, J. Reed, R. T. Moore, J. K. Lucek, and A. D. Ellis, "Low polarization sensitivity electroabsorption modulators for $160 \mathrm{Gbit} / \mathrm{s}$ networks," Electron. Lett., vol. 33, no. 24, pp. 2068-2070, 1997.

[26] J. Tersoff, "Theory of semiconductor heterojunctions: The role of quantum dipoles," Phys. Rev. B, vol. 30, no. 8, pp. 4874-4877, 1984

[27] M. Beaudoin, P. Desjardins, A. Ait-Ouali, J. L. Brebner, R. Y.-F. Yip, H. Marchand, L. Isnard, and R. A. Masut, "Optical properties and heterojunction band alignment in fully coherent strain-compensated $\mathrm{InGa}_{x} \mathrm{P}_{1-x} / \mathrm{Ga}_{y} \mathrm{In}_{1-y} \mathrm{P}$ multilayers on $\operatorname{InP}(001)$," J. Appl. Phys., vol. 87, no. 5, pp. 2320-2326, 2000.

[28] F. Devaux, S. Chelles, A. Ougazzaden, A. Mircea, and J. C. Harmand, "Electroabsorption modulators for high-bit-rate optical communications: A comparison of strained InGaAs/InAlAs and InGaAs/InGaAsP MQW," Semicond. Sci. Technol., vol. 10, no. 7, pp. 887-902, 1995.

[29] J. Minch, S. H. Park, T. Keating, and S. L. Chuang, "Theory and experiment of $\operatorname{In}_{1-x} \mathrm{Ga}_{x} \mathrm{As}_{y} \mathrm{P}_{1-y}$ and $\mathrm{In}_{1-x-y} \mathrm{Ga}_{x} \mathrm{Al}_{y}$ As long-wavelength strained quantum-well lasers," IEEE J. Quantum Electron., vol. 35, pp. 771-782, May 1999.

[30] R. Y.-F. Yip, P. Desjardins, L. Isnard, A. Ait-Ouali, A. Bensaada, H. Marchand, J. L. Brebner, J. F. Currie, and R. A. Masut, "Band alignment and barrier height considerations for the quantum-confined Stark effect," J. Vac. Sci. Technol. A, vol. 16, no. 2, pp. 801-4, 1998.

[31] M. Suzuki, H. Tanaka, and S. Akiba, "Effect of hole pile-up at heterointerface on modulation voltage in GaInAsP electroabsorption modulators," Electron. Lett., vol. 25, no. 2, pp. 88-89, 1989.

[32] T. H. Wood, J. Z. Pastalan, C. A. Burrus Jr, B. C. Johnson, B. I. Miller, J. L. deMiguel, U. Koren, and M. G. Young, "Electric field screening by photogenerated holes in multiple quantum wells: A new mechanism for absorption saturation," Appl. Phys. Lett., vol. 57, no. 11, pp. 1081-3, 1990.
[33] M. K. Chin, S. Niki, H. H. Wieder, and W. S. C. Chang, "Carrier screening of electric field and electroabsorption saturation in InGaAs-GaAs quantum well structure," Electron. Lett., vol. 27, no. 25, pp. 2310-2312, 1991.

Sune Højfeldt received the M.Sc. degree in physics from University of Copenhagen, Copenhagen, Denmark, in 1998 and the Ph.D. degree from the Technical University of Denmark in 2002. His doctoral work focused on modeling of electroabsorption modulators.

From 1996 to 1997, he visited the University of British Columbia, Canada, where he worked on compensating fiber dispersion using patterned III-V semiconductor waveguide structures. Current interests include modeling of carrier dynamics in electroabsorption modulators and modeling of electroabsorption modulators for all-optical applications in high-speed optical communication systems.

Jesper Mørk received the M.Sc. and Ph.D. degrees in electrical engineering from the Technical University of Denmark in 1986 and 1988, respectively.

From 1988 to 1996, he was with Tele Danmark Research. After half a year with Ericsson in Denmark, he switched to the Technical University of Denmark, first with Mikroelektronik Centret and since 1998 with COM, where he is now a Professor and head of the theory and modeling group. His research interests are ultrafast dynamics in semiconductor optical devices, quantum dot devices, all-optical switching, and nonlinear laser dynamics. 